\title{
A coincidence-point problem of Perov type on rectangular cone metric spaces
}

\author{
Fairouz Tchier ${ }^{\mathrm{a}}$, Calogero Vetro ${ }^{\mathrm{b}, *}$, Francesca Vetroc $^{\mathrm{c}}$ \\ ${ }^{a}$ Mathematics Department College of Science (Malaz), King Saud University, P. O. Box 22452, Riyadh, King Saudi Arabia. \\ ${ }^{b}$ Department of Mathematics and Computer Science, University of Palermo, Via Archirafi 34, 90123 Palermo, Italy. \\ ${ }^{c}$ Department of Energy, Information Engineering and Mathematical Models (DEIM), University of Palermo, Viale delle Scienze, 90128 \\ Palermo, Italy.
}

Communicated by C. Alaca

\begin{abstract}
We consider a coincidence-point problem in the setting of rectangular cone metric spaces. Using $\alpha$-admissible mappings and following Perov's approach, we establish some existence and uniqueness results for two self-mappings. Under a compatibility assumption, we also solve a common fixed-point problem. (C)2017 All rights reserved.
\end{abstract}

Keywords: Rectangular cone metric space, spectral radius, solid cone, g-contraction of Perov type, $\alpha$-admissible mapping, $\alpha$-g-contraction of Perov type.

2010 MSC: 47H10, 54H25.

\section{Introduction}

The problem of computing the distance between objects is crucial in the applied sciences. Now, in dealing with such kind of problem, the theory of metric spaces and their generalizations plays a key role in defining the distances between two objects (for example, points, functions or sets). Among the generalizations of metric spaces, we recall rectangular metric spaces (Branciari [7]), K-metric spaces (Zabrejko [27]), cone metric spaces (Huang-Zhang [11]), and rectangular cone metric spaces (Azam-Arshad-Beg [5]), which are useful concepts to define distances in a more general way.

On the other hand, the fixed-point theory is strongly related to the theory of metric spaces (Banach [6]). In fact, many fundamental existence and uniqueness fixed-point results are established in metric spaces and further extended to their generalizations. Fixed-point problems were studied by Abbas-RakočevićIqbal [1, 2], Ahmad-Arshad-Vetro [3], Di Bari-Vetro [10], Kadelburg-Radenović [12, 13], La Rosa-Vetro [15], Samet [19], Vetro [24]. For other results in the setting of rectangular cone metric spaces see also Malhotra-Shukla-Sen [16] and Shukla [21].

Based on the above literature, we investigate the existence of coincidence points and common fixed points for two self-mappings defined on a cone rectangular metric space. Precisely, we use Perov's approach (Perov [17], Perov-Kibenko [18]) and other technical hypotheses.

\footnotetext{
${ }^{*}$ Corresponding author

Email addresses: ftchier@ksu.edu.sa (Fairouz Tchier), calogero.vetro@unipa.it (Calogero Vetro), francesca.vetro@unipa.it (Francesca Vetro)
}

doi:10.22436/jnsa.010.08.25 


\section{Preliminaries}

Two basic books for this study are Aliprantis-Tourky [4], and Deimling [9]. Let $(E,\|\cdot\|)$ be a real Banach space, whose null element is denoted by $\theta$. So $C \subset E$ is called a cone if the following conditions are satisfied:

(a) $C$ is closed, non-empty and $C \neq\{\theta\}$;

(b) $a u+b v \in C$ for each $a, b \in \mathbb{R}$ with $a, b \geqslant 0$ and for each $u, v \in C$;

(c) $\mathrm{C} \cap(-\mathrm{C})=\{\theta\}$.

Given a cone $C \subset E$, we define in $E$ a partial order $\preceq$ with respect to $C$ in the following way: $u \preceq v$ if and only if $v-u \in C$. If $C$ is a solid cone, that is, $\operatorname{Int}(C) \neq \emptyset($ where $\operatorname{Int}(C)$ is the interior of $C)$, then by $u \ll v$ we denote the fact that $v-u \in \operatorname{Int} C$. A cone $C$ is said to be normal if there exists a number $K>0$ such that, for all $u, v \in E$, we have

$$
\theta \preceq u \preceq v \text { implies }\|\mathrm{u}\| \leqslant \mathrm{K}\|v\| \text {. }
$$

The least positive number satisfying the above inequality is called the normal constant of $\mathrm{C}$.

Definition 2.1. Let $(E,\|\cdot\|)$ be a real Banach space, $C$ be a cone in $E$ and let $X$ be a non-empty set. A mapping $\mathrm{d}: \mathrm{X} \times \mathrm{X} \rightarrow \mathrm{C}$ that satisfies the following conditions:

(d1) $d(u, v)=\theta$ if and only if $u=v$;

(d2) $d(u, v)=d(v, u)$ for all $u, v \in X$;

(d3) $d(u, v) \preceq d(u, z)+d(z, w)+d(w, v)$ for all $u, v \in X$ and for all distinct points $w, z \in X \backslash\{u, v\}$;

is called a rectangular cone metric on $\mathrm{X}$ and $(\mathrm{X}, \mathrm{d})$ is called a rectangular cone metric space over the cone $\mathrm{C}$.

We notice that the concept of rectangular cone metric space is more general than the one of rectangular metric space.

Definition 2.2. Let $(X, d)$ be a rectangular cone metric space over a solid cone $C,\left\{u_{n}\right\}$ be a sequence in $X$. Then

(i) $\left\{u_{n}\right\}$ is a Cauchy sequence if, for every $c \in \operatorname{Int}(C)$, there is $n(c) \in \mathbb{N}$ such that, for all $n, m \geqslant n(c)$, $\mathrm{d}\left(\mathrm{u}_{\mathrm{n}}, \mathrm{u}_{\mathrm{m}}\right) \ll \mathrm{c}$;

(ii) $\left\{u_{n}\right\}$ is a convergent sequence if, for every $c \in \operatorname{Int}(C)$, there is $n(c) \in \mathbb{N}$ such that, for all $n \geqslant n(c)$, $d\left(u_{n}, u\right) \ll c$ for some $u \in X$.

We mention that by $u_{n} \stackrel{c}{\rightarrow} u$ we denote the convergence of a sequence $\left\{u_{n}\right\}$ to a limit $u$. So a rectangular cone metric space $(X, d)$ is said to be complete if every Cauchy sequence in $X$ is convergent in $X$. If $C$ is a normal solid cone, then $u_{n} \stackrel{c}{\rightarrow} u$ if and only if $d\left(u_{n}, u\right) \rightarrow \theta$ as $n \rightarrow+\infty$ and $\left\{u_{n}\right\}$ is a Cauchy sequence if and only if $d\left(u_{n}, u_{m}\right) \rightarrow \theta$ as $n, m \rightarrow+\infty$ (see Huang-Zhang [11], Lemmas 1 and 4).

Example 2.3. Let $E=\mathbb{R}^{2}$ and $C=\left\{u=\left(u_{1}, u_{2}\right) \in E: u_{j} \geqslant 0\right.$ for $\left.j=1,2\right\}$. Clearly, $C$ is a normal solid cone with normal constant $K=1$. If $X=\mathbb{N}$ and $d(u, u)=(0,0)$ for all $u \in X, d(2,3)=d(3,2)=(5,11)$ and $d(u, v)=(2,4)$ otherwise, then $d$ is a rectangular cone metric on $X$. Clearly, $(X, d)$ is not a cone metric space because it lacks the triangle property. In fact, we have

$$
\mathrm{d}(2,3)=(5,11) \succeq(2,4)+(2,4)=\mathrm{d}(2,5)+\mathrm{d}(5,3) .
$$

Lemma 2.4. Let $(\mathrm{E},\|\cdot\|)$ be a real Banach space and $\mathrm{C} \subset \mathrm{E}$ be a solid cone. Let $v, w, z \in \mathrm{E}$ and $\left\{\mathrm{a}_{\mathrm{n}}\right\} \subset \mathrm{E}$. Then we have the following properties: 
(j) if $z \preceq w$ and $w \ll v$, then $z \ll v$;

(ji) if $\theta \preceq z \ll c$ for each $c \in \operatorname{Int}(C)$, then $z=\theta$;

(jij) if $\mathrm{c} \in \operatorname{Int}(\mathrm{C})$ and $\mathrm{a}_{\mathrm{n}} \rightarrow \theta$ as $\mathrm{n} \rightarrow+\infty$, then there exists $\mathrm{n}(\mathrm{c})$ such that for all $\mathrm{n} \geqslant \mathrm{n}(\mathrm{c})$ we have $\mathrm{a}_{\mathrm{n}} \ll \mathrm{c}$.

Proof. Here, we prove only (jj). By using Proposition (2.2) at page 20 of [25], we have that $[-\mathrm{c}, \mathrm{c}]=$ $(C-c) \cap(c-C)$ is a neighbourhood of $\theta$ and so also $\operatorname{Int}[-c, c]$. Since $a_{n} \rightarrow \theta$ as $n \rightarrow+\infty$, there exists $n(c)$ such that $a_{n} \in \operatorname{Int}[-c, c]=(\operatorname{Int} C-c) \cap(c-\operatorname{IntC})$ whenever $n \geqslant n(c)$. Now, from $a_{n} \in c-\operatorname{Int}(C)$ (that is, $\left.c-a_{n} \in \operatorname{Int}(C)\right)$ it follows $a_{n} \ll c$ whenever $n \geqslant n(c)$.

Definition 2.5 (Xu-Dolićanin-Radenović [26]). Let $(E,\|\cdot\|)$ be a real Banach space with a solid cone $C$ and let $\left\{u_{n}\right\} \subset C$ be a sequence. We say that $\left\{u_{n}\right\}$ is a c-sequence if, for every $c \in \operatorname{Int}(C)$, there exists $n(c) \in \mathbb{N}$ such that $u_{n} \ll c$ for all $n \geqslant n(c)$.

Remark 2.6. Let $(X, d)$ be a rectangular cone metric space over a solid cone $C,\left\{u_{n}\right\} \subset X$ and $u \in X$. Then

(i) the sequence $\left\{u_{n}\right\}$ converges to $u$ if and only if $\left\{d\left(u_{n}, u\right)\right\}$ is a c-sequence;

(ii) if there exists a c-sequence $\left\{v_{n}\right\}$ such that $d\left(u_{m}, u_{n}\right) \preceq v_{m}$ for all $m \in \mathbb{N}$ and $n \geqslant m$, then $\left\{u_{n}\right\}$ is a Cauchy sequence.

Remark 2.7. Let $C$ be a solid cone in a real Banach space $(E,\|\cdot\|)$ and $\left\{u_{n}\right\}$ be a sequence in $C$. Then the following conditions are equivalent

(i) $\left\{u_{n}\right\}$ is a c-sequence;

(ii) there exists a sequence $\left\{v_{n}\right\} \subset \operatorname{Int}(C)$ such that $v_{n} \rightarrow \theta$ as $n \rightarrow+\infty$, and for any $n \in \mathbb{N}$, there exists $k(n) \in \mathbb{N}$ such that $u_{m} \preceq v_{n}$ for each $m \geqslant k(n)$.

Proof. (i) $\Rightarrow$ (ii). We choose $v_{n}=c / n$ for all $n \in \mathbb{N}$, where $c \in \operatorname{Int}(C)$ is fixed.

(ii) $\Rightarrow$ (i). We use (jjj) of Lemma 2.4 .

\section{Main results}

In this section we prove our main results. We start with some auxiliary notions and results. Let $X$ be a non-empty set, and $f, g: X \rightarrow X$ be mappings such that $f X \subset g X$. Let $u_{0} \in X$ be arbitrary and let $\left\{f u_{n}\right\}$ be the sequence defined by $g u_{n}=f u_{n-1}$ for all $n \in \mathbb{N}$. Moreover, we assume that if $f u_{n}=f u_{n+p}$, for some $p \in \mathbb{N}$, then we choose $u_{n+p+1}=u_{n+1}$. This can be done, since $f X \subset g X$. Here, we call $\left\{f u_{n}\right\}$ a $\mathrm{f}$-g-sequence starting at $u_{0}$ (Vetro [23]). We say that the mapping $f$ is $g$-continuous if $f u_{n} \stackrel{c}{\rightarrow} f u$ whenever $g u_{n} \stackrel{c}{\rightarrow} g u$ for all $u \in X$. Also, if $\xi=f u=g u$ for some $u \in X$, then $u$ is a coincidence point of $f$ and $g$, and $\xi$ is a point of coincidence of $f$ and $g$. Moreover, if $\xi=f \xi=g \xi$, then $\xi$ is a common fixed point of $f$ and $g$. Finally, if $f g v=g f v$, whenever $f v=g v$ for some $v \in X$, then $f$ and $g$ are weakly compatible.

Let $(E,\|\cdot\|)$ be a real Banach space. We write $\mathcal{B}(E)$ for the set of all bounded linear operators on $E$ and $\mathcal{L}(E)$ for the set of all linear operators on $E . \mathcal{B}(E)$ is a Banach algebra. If $A \in \mathcal{B}(E)$, we denote by

$$
\rho(A)=\lim \left\|A^{n}\right\|^{1 / n}=\inf \left\|A^{n}\right\|^{1 / n}
$$

the spectral radius of $A$. Let us remark that if $\rho(A)<1$, the series $\sum_{n=0}^{+\infty} A^{n}$ is absolutely convergent, consequently, $\left\|A^{n}\right\| \rightarrow 0$ as $n \rightarrow+\infty$. Further, $I-A$ is invertible in $\mathcal{B}(E)$ and

$$
\sum_{n=0}^{+\infty} A^{n}=(I-A)^{-1} \text {. }
$$

Moreover, if $\|A\|<1$ then $\rho(A)<1$ and again $I-A$ is invertible and

$$
\left\|(I-A)^{-1}\right\| \leqslant \frac{1}{1-\|A\|} \quad \text { as well as } \quad \rho\left((I-A)^{-1}\right) \leqslant \frac{1}{1-\rho(A)} .
$$

Also, we have that $(I-A)^{-1}$ is nondecreasing if $A$ is nondecreasing. 
Lemma 3.1 (Cvetković-Rakočević [8], Lemma 3.2). Let $(\mathrm{E},\|\cdot\|)$ be a real Banach space, $\mathrm{C} \subset \mathrm{E}$ be a cone and let $A \in \mathcal{L}(\mathrm{E})$. The following conditions are equivalent:

(i) $A$ is nondecreasing, that is, $u \preceq v$ implies $A(u) \preceq A(v)$;

(ii) $\mathrm{A}$ is positive, that is, $\mathrm{A}(\mathrm{C}) \subset \mathrm{C}$.

Proof. (i) $\Rightarrow$ (ii). If $A$ is nondecreasing, then for each $c \in C$, we have $\theta=A(\theta) \preceq A(c)$. Thus, $A(C) \subset C$. (ii) $\Rightarrow$ (i). Let $u, v \in E$ such that $u \preceq v$. This implies $v-u \in C$ and so $A(v-u) \in C$, that is, $A(u) \preceq A(v)$.

For the following result see Lemma 2 of Shukla-Balasubramanian-Pavlović [22].

Lemma 3.2. Let $(E,\|\cdot\|)$ be a real Banach space and $C \subset E$ be a solid cone. Let $A \in \mathcal{L}(E)$ be such that $A(C) \subset C$ and $\rho(A)<1$. Then the following properties hold:

(i) if $\mathrm{a} \in \mathrm{C}$ is such that $\mathrm{a} \preceq A(\mathrm{a})$, then $\mathrm{a}=\theta$;

(ii) $\rho\left(A^{m}\right)<1$ for any fixed $m \in \mathbb{N}$.

Proof. (i). We notice that $(I-A)^{-1}$ exists and, further, $(I-A)^{-1}$ is nondecreasing. Then, from $(I-A)(a) \preceq$ $\theta$, it follows $a=(I-A)^{-1}(I-A)(a) \preceq(I-A)^{-1}(\theta)=\theta$. Taking into account that $a \in C$, we have $a=\theta$.

(ii). Since $\rho(A)<1$, we have

$$
\begin{aligned}
\rho\left(A^{m}\right)=\lim _{n \rightarrow+\infty}\left\|\left(A^{m}\right)^{n}\right\|^{\frac{1}{n}} & =\lim _{n \rightarrow+\infty}\left\|\left(A^{n}\right)^{m}\right\|^{\frac{1}{n}} \\
& \leqslant \lim _{n \rightarrow+\infty}\left(\left\|A^{n}\right\|^{\frac{1}{n}}\right)^{m}=\left(\lim _{n \rightarrow+\infty}\left\|A^{n}\right\|^{\frac{1}{n}}\right)^{m}=(\rho(A))^{m}<1 .
\end{aligned}
$$

Lemma 3.3. Let $(\mathrm{E},\|\cdot\|)$ be a real Banach space, $\mathrm{C} \subset \mathrm{E}$ be a solid cone, $\mathrm{A} \in \mathcal{B}(\mathrm{E})$ be a nondecreasing operator and $\left\{\mathrm{u}_{\mathrm{n}}\right\} \subset \mathrm{C}$ be a c-sequence. Then $\mathrm{A}\left(\mathrm{u}_{\mathrm{n}}\right)$ is a c-sequence.

Proof. By Remark 2.7, there exists a sequence $\left\{v_{n}\right\} \subset \operatorname{Int}(\mathrm{C})$ with $v_{\mathrm{n}} \rightarrow \theta$ as $\mathrm{n} \rightarrow+\infty$, and $k(\mathrm{n}) \in \mathbb{N}$ such that $u_{m} \preceq v_{n}$ for each $m \geqslant k(n)$. Thus $A\left(u_{m}\right) \preceq A\left(v_{n}\right)$ for each $m \geqslant k(n)$. Since $A\left(v_{n}\right) \rightarrow \theta$ as $n \rightarrow+\infty$, by (jjj) of Lemma 2.4, it follows that $A\left(v_{n}\right)$ is a c-sequence and, consequently, $A\left(u_{n}\right)$ is a c-sequence.

Lemma 3.4. Let $(X, d)$ be a rectangular cone metric space and $\left\{u_{n}\right\} \subset X$ be a sequence such that

(i) $\left\{\mathrm{d}\left(\mathrm{u}_{\mathrm{n}}, \mathrm{u}_{\mathrm{n}+1}\right)\right\}$ is a c-sequence;

(ii) $u_{n} \neq u_{m}$ whenever $n \neq m$;

(ii) $u, v \notin\left\{u_{n}: n \in \mathbb{N}\right\}$.

If $\left\{u_{n}\right\}$ converges to both $u$ and $v$, then $u=v$.

Proof. From

$$
d(u, v) \preceq d\left(u, u_{n}\right)+d\left(u_{n}, u_{n+1}\right)+d\left(u_{n+1}, v\right),
$$

taking into account that $\left\{d\left(u, u_{n}\right)+d\left(u_{n}, u_{n+1}\right)+d\left(u_{n+1}, v\right)\right\}$ is a c-sequence, by using (jj) of Lemma 2.4, we deduce $d(u, v)=\theta$, that is, $u=v$.

Now, we give the definition of $g$-contraction of Perov type in the framework of rectangular cone metric spaces over solid cone.

Definition 3.5. Let $(X, d)$ be a rectangular cone metric space and let $f, g: X \rightarrow X$ be two mappings. $f$ is called a $g$-contraction of Perov type if there exists $A \in \mathcal{B}(E)$ with $\rho(A)<1$ such that

$$
d(f u, f v) \preceq A(d(g u, g v)) \text { for all } u, v \in X .
$$


Theorem 3.6. Let $(\mathrm{X}, \mathrm{d})$ be a rectangular cone metric space over a solid cone $\mathrm{C}$ and let $\mathrm{f}, \mathrm{g}: \mathrm{X} \rightarrow \mathrm{X}$ be such that $\mathrm{fX} \subset \mathrm{gX}$. If $\mathrm{f}$ is a g-contraction of Perov type with $\mathrm{A}(\mathrm{C}) \subset \mathrm{C}$ and at least one of $\mathrm{fX}$ or $\mathrm{gX}$ is a complete subset of $\mathrm{X}$, then $\mathrm{f}$ and $\mathrm{g}$ have a unique point of coincidence in $\mathrm{X}$. Moreover, if $\mathrm{f}$ and $\mathrm{g}$ are weakly compatible, then they have a unique common fixed point.

Proof. Let $u_{0} \in X$ and $\left\{f u_{n}\right\}$ be a f-g-sequence starting at $u_{0}$. If $f u_{k-1}=f u_{k}$ for some $k \in \mathbb{N}$, from fut $f u_{k-1}=g u_{k}$, we get that $f u_{k}$ is a point of coincidence of $f$ and $g$. Therefore, we can suppose $f u_{n-1} \neq f u_{n}$ for all $n \in \mathbb{N}$. First, we prove that $f u_{n} \neq f u_{m}$ for all $n, m \in \mathbb{N}$ with $n \neq m$. Proceeding by contradiction, suppose that there exist $n, p \in \mathbb{N}$ such that $f u_{n}=f u_{n+p}$ with $p \geqslant 2$. Then $f u_{n+1}=f u_{n+p+1}$. Now, using (3.1), we obtain

$$
d\left(f u_{n}, f u_{n+1}\right)=d\left(f u_{n+p}, f u_{n+p+1}\right) \preceq A\left(d\left(g u_{n+p}, g u_{n+p+1}\right)\right)=A\left(d\left(f u_{n+p-1}, f u_{n+p}\right)\right) .
$$

From the previous inequality, we get

$$
d\left(f u_{n}, f u_{n+1}\right) \preceq A^{p}\left(d\left(f u_{n}, f u_{n+1}\right)\right) .
$$

Further, using Lemma 3.2, we get $d\left(f u_{n}, f u_{n+1}\right)=\theta$ which is a contradiction since $f u_{n} \neq f u_{n+1}$. Thus $f u_{n} \neq f u_{m}$ for all $n, m \in \mathbb{N}$ with $n \neq m$.

Now, we show that $\left\{f u_{n}\right\}$ is a Cauchy sequence. Using (3.1), with $u=u_{n}$ and $v=u_{n+1}$, we get

$$
d\left(f u_{n}, f u_{n+1}\right) \preceq A\left(d\left(g u_{n}, g u_{n+1}\right)\right)=A\left(d\left(f u_{n-1}, f u_{n}\right)\right) \text { for all } n \in \mathbb{N} .
$$

Again, using (3.1) with $u=u_{n}$ and $v=u_{n+2}$, we have

$$
d\left(f u_{n}, f u_{n+2}\right) \preceq A\left(d\left(g u_{n}, g u_{n+2}\right)\right)=A\left(d\left(f u_{n-1}, f u_{n+1}\right)\right) \text { for all } n \in \mathbb{N} .
$$

Since $A$ is nondecreasing, from the previous inequalities, we obtain

$$
d\left(f u_{n}, f u_{n+1}\right) \preceq A^{n}\left(d\left(f u_{0}, f u_{1}\right)\right) \quad \text { and } \quad d\left(f u_{n}, f u_{n+2}\right) \preceq A^{n}\left(d\left(f u_{0}, f u_{2}\right)\right) \text { for all } n \in \mathbb{N} \text {. }
$$

Let $n, m \in \mathbb{N}$ with $n>m$. If $n-m$ is odd, by using the rectangular inequality, we get

$$
\begin{aligned}
d\left(f u_{m}, f u_{n}\right) & \preceq d\left(f u_{m}, f u_{m+1}\right)+d\left(f u_{m+1}, f u_{m+2}\right)+\cdots+d\left(f u_{n-1}, f u_{n}\right) \\
& \preceq\left(A^{m}+A^{m+1}+\cdots+A^{n-1}\right)\left(d\left(f u_{0}, f u_{1}\right)\right) \\
& \preceq A^{m}(I-A)^{-1}\left(d\left(f u_{0}, f u_{1}\right)\right) .
\end{aligned}
$$

From $\left\|A^{m}(I-A)^{-1}\left(d\left(f u_{0}, f u_{1}\right)\right)\right\| \leqslant\left\|A^{m}\right\|\left\|(I-A)^{-1}\left(d\left(f u_{0}, f u_{1}\right)\right)\right\| \rightarrow 0$ for $m \rightarrow+\infty$, we deduce that $A^{m}\left((I-A)^{-1}\left(d\left(f u_{0}, f u_{1}\right)\right)\right) \rightarrow \theta$ as $m \rightarrow+\infty$. Therefore, by (jj) of Lemma 2.4, we have that the sequence $\left\{A^{m}\left((I-A)^{-1}\left(d\left(f u_{0}, f u_{1}\right)\right)\right)\right\}$ is a c-sequence and so, by (ii) of Remark 2.6, we conclude that $\left\{f u_{n}\right\}$ is a Cauchy sequence.

If $n-m$ is even, by the rectangular inequality, we have

$$
\begin{aligned}
d\left(f u_{m}, f u_{n}\right) & \preceq d\left(f u_{m}, f u_{m+1}\right)+\cdots+d\left(f u_{n-3}, f u_{n-2}\right)+d\left(f u_{n-2}, f u_{n}\right) \\
& \preceq\left(A^{m}+A^{m+1}+\cdots+A^{n-3}\right)\left(d\left(f u_{0}, f u_{1}\right)\right)+A^{n-2}\left(d\left(f u_{0}, f u_{2}\right)\right) \\
& \preceq A^{m}(I-A)^{-1}\left(d\left(f u_{0}, f u_{1}\right)\right)+A^{n-2}\left(d\left(f u_{0}, f u_{2}\right)\right) .
\end{aligned}
$$

Consequently, we get that $\left\{f u_{n}\right\}$ is a Cauchy sequence. Taking into account that $f X \subset g X$ and one between $f X$ and $g X$ is complete, we deduce that the sequence $\left\{f u_{n}\right\}$ converges to a point $\xi=g \zeta \in g X$. Now we show that $f \zeta=\xi$. If $f \zeta \neq \xi$, from $f u_{n} \neq f u_{m}$ whenever $n \neq m$, we get that there exists $n(\xi) \in \mathbb{N}$ such that

$$
\xi \neq f u_{n} \neq f u_{n+1} \neq f \zeta \text { for all } n \geqslant n(\xi) .
$$


Thus, for all $n \geqslant n(\xi)$, we have

$$
\begin{aligned}
d(\xi, f \zeta) & \preceq d\left(\xi, f u_{n}\right)+d\left(f u_{n}, f u_{n+1}\right)+d\left(f u_{n+1}, f \zeta\right) \\
& \preceq d\left(\xi, f u_{n}\right)+d\left(f u_{n}, f u_{n+1}\right)+A\left(d\left(g u_{n+1}, g \zeta\right)\right) .
\end{aligned}
$$

Taking into account that, by Lemma 3.3, $d\left(\xi, f u_{n}\right)+d\left(f u_{n}, f u_{n+1}\right)+A\left(d\left(g u_{n+1}, g \zeta\right)\right.$ is a c-sequence, by (3.2) we deduce that $d(\xi, f \zeta)=\theta$, that is, $\xi=f \zeta$ and hence $\xi$ is a point of coincidence of $f$ and $g$.

Now, we must only prove that $f$ and $g$ have a unique point of coincidence in $X$. We suppose that $v \neq \xi$ is another point of coincidence. This implies that there is $\omega \in X$ with $f \omega=g \omega=v$. Hence, we have

$$
d(\xi, v)=d(f \zeta, f \omega) \preceq A(d(g \xi, g \omega))=A(d(\xi, v)) .
$$

Using (i) of Lemma 3.2, we deduce that $\xi=v$, that is, $f$ and $g$ have a unique point of coincidence. Taking into account that $\xi$ is the unique point of coincidence of $f$ and $g$ and, further, $f$ and $g$ are weakly compatible that implies $f \xi$ is a point of coincidence, since $f \xi=f g \zeta=g f \zeta=g \xi$, we get $\xi=f \xi=g \xi$. Thus $\xi$ is the unique common fixed point of $f$ and $g$.

\section{4. $\alpha$-contraction of Perov type}

In this section, we consider $\alpha$-admissible mappings (see Kumam-Vetro-Vetro [14], Samet-Vetro-Vetro [20]) and introduce the notion of $\alpha$-g-contraction of Perov type in the setting of rectangular cone metric spaces. For this class of contractions, we give again results of existence and uniqueness of points of coincidence and common fixed points. In the sequel $(E,\|\cdot\|)$ is a real Banach space.

Definition 4.1. Let $(X, d)$ be a rectangular cone metric space and let $f, g: X \rightarrow X$ be two mappings. $f$ is called an $\alpha$-g-contraction of Perov type if there exist a function $\alpha: X \times X \rightarrow[0,+\infty)$ and $A \in \mathcal{B}(E)$ with $\rho(A)<1$ such that

$$
d(f u, f v) \preceq A(d(g u, g v))
$$

for all $u, v \in X$ with $\alpha(g u, g v) \geqslant 1$.

Remark 4.2. Let $f, g: X \rightarrow X$ be two mappings. If $f: X \rightarrow X$ is a $g$-contraction of Perov type, then $f$ is an $\alpha$-g-contraction of Perov type where $\alpha(u, v)=1$ for all $u, v \in X$.

Definition 4.3. Let $f, g: X \rightarrow X$ and $\alpha: X \times X \rightarrow[0,+\infty)$. We say that $f$ is $\alpha$-g-admissible if

$$
\text { for } u, v \in X \text { with } g u \neq g v, \quad \alpha(g u, g v) \geqslant 1 \quad \text { implies } \quad \alpha(f u, f v) \geqslant 1 .
$$

Example 4.4. Let $X=(0,+\infty)$. Define $f, g: X \rightarrow X$ and $\alpha: X \times X \rightarrow[0,+\infty)$ as follows

$$
f u=e^{u}+\ln u, \quad g u=2 u \quad \text { for all } u \in X,
$$

and, for $u, v \in X$,

$$
\alpha(u, v)= \begin{cases}3, & \text { if } u \geqslant v \\ 0, & \text { if } u<v .\end{cases}
$$

Then, $f$ is $\alpha$-g-admissible.

Our first result is the following theorem for a g-continuous mapping.

Theorem 4.5. Let $(\mathrm{X}, \mathrm{d})$ be a rectangular cone metric space over a solid cone $\mathrm{C}$ and let $\mathrm{f}, \mathrm{g}: \mathrm{X} \rightarrow \mathrm{X}$ be such that $\mathrm{fX} \subset \mathrm{gX}$ and at least one of $\mathrm{fX}$ or $\mathrm{gX}$ is a complete subset of $\mathrm{X}$. Suppose that $\mathrm{f}$ is an $\alpha-\mathrm{g}$-contraction of Perov type with respect to one function $\alpha: X \times X \rightarrow[0,+\infty)$ and $\mathrm{A}(\mathrm{C}) \subset \mathrm{C}$. Assume that the following conditions hold:

(i) there exists $\mathrm{u}_{0} \in X$ such that $\alpha\left(g u_{0}, f u_{0}\right) \geqslant 1$; 
(ii) $f$ is $\alpha$-g-admissible;

(iii) if $\left\{u_{n}\right\} \subset X$ is a sequence such that $\alpha\left(g u_{n}, g u_{n+1}\right) \geqslant 1$ for all $n \in \mathbb{N}$, then $\alpha\left(g u_{n}, g u_{n+2}\right) \geqslant 1$ for all $\mathrm{n} \in \mathbb{N}$;

(iv) $\mathrm{f}$ is g-continuous.

Then $\mathrm{f}$ and $\mathrm{g}$ have a point of coincidence in $\mathrm{X}$.

Proof. Let $\mathfrak{u}_{0} \in X$ be such that $\alpha\left(g \mathfrak{u}_{0}, f \mathfrak{u}_{0}\right) \geqslant 1$ (see (i)) and let $\left\{f u_{n}\right\}$ be a $f$-g-sequence starting at $\mathfrak{u}_{0}$. If $f u_{k-1}=f u_{k}$ for some $k \in \mathbb{N}$, from $f u_{k}=f u_{k-1}=g u_{k}$, we get that $f u_{k}$ is a point of coincidence of $f$ and g. Therefore, we can suppose $f u_{n-1} \neq f u_{n}$ for all $n \in \mathbb{N}$. It is important to note that conditions (i) and (ii) ensure that

$$
\alpha\left(g u_{n}, g u_{n+1}\right) \geqslant 1 \text { for all } n \in \mathbb{N} .
$$

First, proceeding as in the proof of Theorem 3.6, using (4.2), we deduce that $f u_{n} \neq f u_{m}$ for all $n, m \in \mathbb{N}$ with $n \neq m$. Next, using (4.1) with $u=u_{n}$ and $v=u_{n+1}$, we get

$$
d\left(f u_{n}, f u_{n+1}\right) \preceq A\left(d\left(g u_{n}, g u_{n+1}\right)\right)=A\left(d\left(f u_{n-1}, f u_{n}\right)\right) \text { for all } n \in \mathbb{N} .
$$

By (iii) and (4.2), we deduce that $\alpha\left(g u_{n}, g u_{n+2}\right) \geqslant 1$ for all $n \in \mathbb{N}$. Thus, using (4.1) with $u=u_{n}$ and $v=u_{n+2}$, we obtain

$$
d\left(f u_{n}, f u_{n+2}\right) \preceq A\left(d\left(g u_{n}, g u_{n+2}\right)\right)=A\left(d\left(f u_{n-1}, f u_{n+1}\right)\right) \text { for all } n \in \mathbb{N} .
$$

Proceeding as in the proof of Theorem 3.6, using (4.3) and (4.4), we deduce that $\left\{f u_{n}\right\}$ is a Cauchy sequence. Taking into account that $f X \subset g X$ and one between $f X$ and $g X$ is complete, we obtain that the sequence $\left\{f u_{n}\right\}$, or equivalently, $\left\{g u_{n}\right\}$ converges to a point $\xi=g \zeta \in g X$. Now, we show that $f \zeta=\xi$. Assume that $f \zeta \neq \xi$. Since $f$ is $g$-continuous and $g u_{n} \stackrel{c}{\rightarrow} g \zeta$, then $f u_{n} \stackrel{c}{\rightarrow} f \zeta$. By Lemma 3.4, from

$$
f u_{\mathrm{n}} \stackrel{\mathrm{c}}{\rightarrow} \mathrm{f} \zeta \quad \text { and } \quad \mathrm{fu} \mathrm{u}_{\mathrm{n}} \stackrel{\mathrm{c}}{\rightarrow} \xi,
$$

we obtain $\xi=f \zeta$, which is a contradiction, and so $\xi$ is a point of coincidence of $f$ and $g$.

In the next theorem, we omit the g-continuity hypothesis of $f$.

Theorem 4.6. Let $(\mathrm{X}, \mathrm{d})$ be a rectangular cone metric space over a solid cone $\mathrm{C}$ and let $\mathrm{f}, \mathrm{g}: \mathrm{X} \rightarrow \mathrm{X}$ be such that $\mathrm{fX} \subset \mathrm{gX}$ and at least one of $\mathrm{fX}$ or $\mathrm{gX}$ is a complete subset of $\mathrm{X}$. Suppose that $\mathrm{f}$ is an $\alpha-\mathrm{g}$-contraction of Perov type with respect to one function $\alpha: X \times X \rightarrow[0,+\infty)$ and $\mathrm{A}(\mathrm{C}) \subset \mathrm{C}$. Assume that the following conditions hold:

(i) there exists $\mathfrak{u}_{0} \in X$ such that $\alpha\left(g u_{0}, \mathrm{fu}_{0}\right) \geqslant 1$;

(ii) $\mathrm{f}$ is $\alpha$-g-admissible;

(iii) if $\left\{u_{n}\right\} \subset X$ is a sequence such that $\alpha\left(g u_{n}, g u_{n+1}\right) \geqslant 1$ for all $n \in \mathbb{N}$, then $\alpha\left(g u_{n}, g u_{n+2}\right) \geqslant 1$ for all $\mathrm{n} \in \mathbb{N}$;

(iv) if $\left\{\mathrm{u}_{\mathrm{n}}\right\}$ is a sequence in $\mathrm{X}$ such that $\alpha\left(\mathrm{gu}, \mathrm{g} \mathrm{u}_{\mathrm{n}+1}\right) \geqslant 1$ for all $\mathrm{n} \in \mathbb{N}$ and $\mathrm{gu} \mathrm{u}_{\mathrm{n}} \stackrel{\mathrm{c}}{\rightarrow} \mathrm{gu} \in \mathrm{X}$ as $\mathrm{n} \rightarrow+\infty$, then $\alpha\left(g u_{n}, g u\right) \geqslant 1$ for all $n \in \mathbb{N}$.

Then $\mathrm{f}$ and $\mathrm{g}$ have a point of coincidence in $\mathrm{X}$.

Proof. Let $\mathrm{u}_{0} \in X$ be such that $\alpha\left(g u_{0}, f u_{0}\right) \geqslant 1$ (see (i)) and let $\left\{f u_{n}\right\}$ be a f-g-sequence starting at $u_{0}$. If $f u_{k-1}=f u_{k}$ for some $k \in \mathbb{N}$, from $f u_{k}=f u_{k-1}=g u_{k}$, we get that $f u_{k}$ is a point of coincidence of $f$ and g. Therefore, we suppose $f u_{n-1} \neq f u_{n}$ for all $n \in \mathbb{N}$. By conditions (i) and (ii) we have

$$
\alpha\left(g u_{n}, g u_{n+1}\right) \geqslant 1 \text { for all } n \in \mathbb{N} .
$$


First, proceeding as in the proof of Theorem 3.6, using (4.5), we deduce that $f u_{n} \neq f u_{m}$ for all $n, m \in \mathbb{N}$ with $n \neq m$. Next, using (4.1) with $u=u_{n}$ and $v=u_{n+1}$, we get

$$
d\left(f u_{n}, f u_{n+1}\right) \preceq A\left(d\left(g u_{n}, g u_{n+1}\right)\right)=A\left(d\left(f u_{n-1}, f u_{n}\right)\right) \text { for all } n \in \mathbb{N} .
$$

By (iii) and (4.5), we deduce that $\alpha\left(g u_{n}, g u_{n+2}\right) \geqslant 1$ for all $n \in \mathbb{N}$. Then, using (4.1) with $u=u_{n}$ and $v=u_{n+2}$, we obtain

$$
d\left(f u_{n}, f u_{n+2}\right) \preceq A\left(d\left(g u_{n}, g u_{n+2}\right)\right)=A\left(d\left(f u_{n-1}, f u_{n+1}\right)\right) \text { for all } n \in \mathbb{N} .
$$

Proceeding as in the proof of Theorem 3.6, using (4.6) and (4.7), we deduce that $\left\{f u_{n}\right\}$ is a Cauchy sequence. Taking into account that $f X \subset g X$ and one between $f X$ and $g X$ is complete, we deduce that the sequence $\left\{f u_{n}\right\}$, or equivalently, $\left\{g u_{n}\right\}$ converges to a point $\xi=g \zeta \in g X$. Now, we show that $f \zeta=\xi$. Assume that $f \zeta \neq \xi$. The hypothesis (iv) ensures that $\alpha\left(g u_{n}, g \zeta\right) \geqslant 1$ for all $n \in \mathbb{N}$. Using (4.1) with $\mathrm{u}=\mathrm{u}_{\mathrm{n}}$ and $v=\zeta$, we obtain

$$
d\left(f u_{n}, f \zeta\right) \preceq A\left(d\left(g u_{n}, g \zeta\right)\right) \text { for all } n \in \mathbb{N} .
$$

Since $\left\{d\left(g u_{n}, g \zeta\right)\right\}$ is a c-sequence, we deduce that $\left\{d\left(f u_{n}, f \zeta\right)\right\}$ is a c-sequence, that is, $f u_{n} \stackrel{c}{\rightarrow} f \zeta$. By Lemma 3.4, from

$$
\mathrm{fu}_{\mathrm{n}} \stackrel{\mathrm{c}}{\rightarrow} \mathrm{f} \zeta \quad \text { and } \quad \mathrm{fu}_{\mathrm{n}} \stackrel{\mathrm{c}}{\rightarrow} \xi,
$$

we obtain $\xi=f \zeta$, which is a contradiction, and hence $\xi$ is a point of coincidence of $f$ and $g$.

In order to assure the uniqueness of the point of coincidence, we will consider the following hypothesis.

$(\mathrm{H})$ : For all $u, v \in X$ with $g u \neq g v$, there exists $z \in X$ such that $\alpha(g u, g z) \geqslant 1, \alpha(g v, g z) \geqslant 1$, and $\alpha(g z, f z) \geqslant 1$

Theorem 4.7. Adding condition $(\mathrm{H})$ to the hypotheses of Theorem 4.5 (resp. Theorem 4.6) we obtain the uniqueness of the point of coincidence of $\mathrm{f}$ and $\mathrm{g}$. Moreover, if $\mathrm{f}$ and $\mathrm{g}$ are weakly compatible, then they have a unique common fixed point.

Proof. Suppose that $\xi$ and $\zeta$ are two points of coincidence of $f$ and $g$ with $\xi \neq \zeta$. Then there exist $u, v \in X$, with $g u \neq g v$, such that $\xi=f u=g u$ and $\zeta=f v=g v$. From $(H)$ there exists $z \in X$ such that $\alpha(g u, g z) \geqslant 1$ and $\alpha(g v, g z) \geqslant 1$. Let $\left\{f z_{n}\right\}$ be a f-g-sequence starting at $z_{0}=z$. From $\alpha\left(g u, g z_{0}\right) \geqslant 1$ and $\alpha\left(g v, g z_{0}\right) \geqslant 1$, using that $f$ is $\alpha$-g-admissible, we have respectively $\alpha\left(f u, f z_{0}\right)=\alpha\left(g u, g z_{1}\right) \geqslant 1$ and $\alpha\left(f v, f z_{0}\right)=\alpha\left(g v, g z_{1}\right) \geqslant 1$. Iterating this process, we obtain

$$
\alpha\left(g u, g z_{n}\right) \geqslant 1 \text { and } \alpha\left(g v, g z_{n}\right) \geqslant 1 \text { for all } n \in \mathbb{N} .
$$

Using (4.1) with $v=z_{n}$, we get

$$
\begin{gathered}
d\left(f u, f z_{\mathfrak{n}}\right) \preceq A\left(d\left(g u, g z_{n}\right)\right)=A\left(d\left(f u, f z_{n-1}\right)\right) \text { for all } n \in \mathbb{N} . \\
d\left(f v, f z_{\mathfrak{n}}\right) \preceq A\left(d\left(g v, g z_{n}\right)\right)=A\left(d\left(f v, f z_{n-1}\right)\right) \text { for all } n \in \mathbb{N} .
\end{gathered}
$$

If $f z_{\mathrm{m}-1}=\mathrm{f} z_{\mathrm{m}}$ for some $\mathrm{m} \in \mathbb{N}$, by (4.8) and (4.9), we have

$$
d\left(f u, f z_{m-1}\right)=d\left(f u, f z_{m}\right) \preceq A\left(d\left(f u, f z_{m-1}\right)\right)
$$

and

$$
d\left(f v, f z_{m-1}\right)=d\left(f v, f z_{m}\right) \preceq A\left(d\left(f v, f z_{m-1}\right)\right),
$$


which imply $f u=f z_{m-1}=f v((i)$ of Lemma 3.2). This is a contradiction, since $f u \neq f v$. Consequently, $\mathrm{f} z_{\mathrm{n}-1} \neq \mathrm{f} z_{\mathrm{n}}$ for all $\mathrm{n} \in \mathbb{N}$. Moreover, $\mathrm{fu} \neq \mathrm{f} z_{\mathrm{n}-1}$ for all $\mathrm{n} \in \mathbb{N}$. In fact, if $\mathrm{fu}=\mathrm{f} z_{\mathrm{n}-1}=g z_{\mathrm{n}}$, from $\alpha(g v, f u)=\alpha\left(g v, g z_{n}\right) \geqslant 1$, using (4.1), we obtain

$$
d(f v, f u) \preceq A(d(g v, g u))=A(d(f v, f u)) .
$$

This implies $f v=f u$ that is a contradiction. Then $f z_{n} \neq f u, f v$ for all $n \in \mathbb{N}$. Since $A$ is nondecreasing, by (4.8) and (4.9), we obtain

$$
d\left(f u, f z_{n}\right) \preceq A^{n}\left(d\left(f u, f z_{0}\right)\right) \text { and } d\left(f v, f z_{n}\right) \preceq A^{n}\left(d\left(f v, f z_{0}\right)\right) \text { for all } n \in \mathbb{N} .
$$

The previous inequalities ensure that $\left\{d\left(f u, f z_{\mathfrak{n}}\right)\right\}$ and $\left\{d\left(f v, f z_{\mathfrak{n}}\right)\right\}$ are c-sequences. Since $z$ satisfies the condition $\alpha(g z, f z) \geqslant 1$, we have that $\left\{d\left(f z_{n}, f z_{n+1}\right)\right\}$ is a c-sequence. Thus from

$$
d(f u, f v) \preceq d\left(f u, f z_{\mathfrak{n}}\right)+d\left(f z_{\mathfrak{n}}, f z_{\mathfrak{n}+1}\right)+d\left(f z_{\mathfrak{n}+1}, f v\right),
$$

we obtain $f u=f v$ that is a contradiction. It follows that $\xi$ is the unique point of coincidence of $f$ and $g$.

Now, suppose that the mappings $f$ and $g$ are weakly compatible. Taking into account that $\xi=f \zeta=g \zeta$ is the unique point of coincidence of $f$ and $g$ and, further, $f$ and $g$ are weakly compatible that implies $f \xi$ is a point of coincidence of $f$ and $g$, since $f \xi=f g \zeta=g f \zeta=g \xi$, we get $\xi=f \xi=g \xi$. Thus $\xi$ is the unique common fixed point of $f$ and $g$.

\section{Applications}

In this section we give two examples of functions $\alpha$ with the properties involved in Theorems 4.5-4.7.

\subsection{Ordered rectangular cone metric space}

Let $(X, d)$ be a rectangular cone metric space over a solid cone $C \subset E$, where $(E,\|\cdot\|)$ is a real Banach space, and let $f, g: X \rightarrow X$ be such that $f X \subset g X$. If $X$ is equipped with a partial order $\precsim$, then $(X, d, \precsim)$ is an ordered rectangular cone metric space. In this case we consider a function $\alpha: X \times X \rightarrow[0,+\infty)$ defined by

$$
\alpha(u, v)= \begin{cases}1, & \text { if } u \precsim v, \\ 0, & \text { otherwise. }\end{cases}
$$

Now, we consider the following conditions:

(a) there exists $u_{0} \in X$ such that $g u_{0} \precsim f u_{0}$;

(b) $f$ is g-nondecreasing, that is, $f u \precsim f v$ whenever $g u \precsim g v$ for all $u, v \in X$;

(c) if $\left\{u_{n}\right\}$ is a sequence in $X$ such that $g u_{n} \precsim g u_{n+1}$ for all $n$ and $g u_{n} \stackrel{c}{\rightarrow} g u \in X$ as $n \rightarrow+\infty$, then $g u_{n} \precsim g u$ for all $n \in \mathbb{N}$.

If condition (a) holds, then the function $\alpha$ given by (5.1) has the property (i) of Theorem 4.6. If (b) holds, then $\alpha$ has the property (ii) of Theorem 4.6. If (c) holds, then $\alpha$ has the property (iv) of Theorem 4.6. Clearly, $\alpha$ always satisfies the property (iii) of Theorem 4.6. Moreover, the function $\alpha$ satisfies condition (H) if the partial order $\precsim$ has the following property:

$(\mathrm{H}(\precsim))$ : for all $u, v \in X$ with $g u \neq g v$, there exists $z \in X$ such that $g u \precsim g z, g v \precsim g z$ and $g z \precsim f z$.

Using Theorems 4.6-4.7, we give the following result in the setting of ordered rectangular cone metric spaces.

Theorem 5.1. Let $(\mathrm{X}, \mathrm{d}, \precsim)$ be an ordered rectangular cone metric space over a solid cone $\mathrm{C}$ and let $\mathrm{f}, \mathrm{g}: \mathrm{X} \rightarrow \mathrm{X}$ be such that $\mathrm{fX} \subset \mathrm{gX}$ and at least one of $\mathrm{fX}$ or $\mathrm{gX}$ is a complete subset of $\mathrm{X}$. Suppose that there exists an operator $A \in \mathcal{B}(E)$ with $\rho(A)<1$ and $A(C) \subset C$ such that

$$
\mathrm{d}(\mathrm{fu}, \mathrm{fv}) \preceq \mathrm{A}(\mathrm{d}(\mathrm{gu}, \mathrm{gv})) \text { for all } \mathrm{u}, v \in \mathrm{X} \text { with } \mathrm{gu} \precsim \mathrm{gv} .
$$

If conditions (a)-(c) hold, then $\mathrm{f}$ and $\mathrm{g}$ have a point of coincidence in $\mathrm{X}$. If also $(H(\precsim))$ holds the point of coincidence is unique. Moreover, if $\mathrm{f}$ and $\mathrm{g}$ are weakly compatible, then they have a unique common fixed point. 


\subsection{Graph rectangular cone metric space}

Definition 5.2. A graph $G$ is an ordered pair $(V, \mathcal{E})$, where $V$ is a set and $\mathcal{E} \subset \mathrm{V} \times \mathrm{V}$ is a binary relation. We say that $V$ is the vertex set and $\mathcal{E}$ is the edge set.

Definition 5.3. Let $G=(V, \mathcal{E})$ be a graph, $D$ be a subset of $V$, and $f, g: V \rightarrow V$ be two mappings. We say that $\mathrm{D}$ is $\mathrm{G}$-directed with respect to $f$ and $g$ if for every $u, v \in \mathrm{D}$ with $\mathrm{gu} \neq \mathrm{g} v$, there exists $z \in \mathrm{V}$ such that $(g u, g z),(g v, g z),(g z, f z) \in \mathcal{E}$.

Definition 5.4. Le $f, g: V \rightarrow V$ be two mappings. We say that $f$ is $g-G$-monotone if $(g u, g v) \in \mathcal{E}$ implies $(f u, f v) \in \mathcal{E}$ for all $u, v \in X$ with $g u \neq g v$.

If $X$ is equipped with a graph $G=(X, \varepsilon)$, then $(X, d, G)$ is a graph rectangular cone metric space. In this case we consider a function $\alpha: X \times X \rightarrow[0,+\infty)$ defined by

$$
\alpha(u, v)= \begin{cases}1, & \text { if }(u, v) \in \mathcal{E}, \\ 0, & \text { otherwise. }\end{cases}
$$

Now, we consider the following conditions:

(a) there exists $u_{0} \in X$ such that $\left(g u_{0}, f u_{0}\right) \in \mathcal{E}$;

(b) $f$ is $g-G$-monotone;

(c) if $\left\{u_{n}\right\} \subset X$ is a sequence such that $\left(g u_{n}, g u_{n+1}\right) \in \mathcal{E}$ for all $n \in \mathbb{N}$, then $\left(g u_{n}, g u_{n+2}\right) \in \mathcal{E}$ for all $\mathrm{n} \in \mathbb{N}$;

(d) if $\left\{u_{n}\right\} \subset X$ is a sequence such that $\left(g u_{n}, g u_{n+1}\right) \in \mathcal{E}$ for all $n \in \mathbb{N}$ and $g u_{n} \stackrel{c}{\rightarrow} g u \in X$ as $n \rightarrow+\infty$, then $\left(g u_{n}, g u\right) \in \mathcal{E}$ for all $n \in \mathbb{N}$.

If condition (a) holds, then the function $\alpha$ given by (5.2) has the property (i) of Theorem 4.6. If (b) holds, then $\alpha$ has the property (ii) of Theorem 4.6. If (c) holds, then $\alpha$ has the property (iii) of Theorem 4.6. Clearly, $\alpha$ has the property (iv) whenever (d) holds. Moreover, the function $\alpha$ satisfies condition (H) if $X$ is $\mathrm{G}$-directed with respect to $\mathrm{f}$ and $\mathrm{g}$.

Using Theorems 4.6-4.7, we deduce the following result in the setting of graph rectangular cone metric spaces.

Theorem 5.5. Let $(\mathrm{X}, \mathrm{d}, \mathrm{G})$ be a graph rectangular cone metric space over a solid cone $\mathrm{C}$ and let $\mathrm{f}, \mathrm{g}: \mathrm{X} \rightarrow \mathrm{X}$ be such that $\mathrm{fX} \subset \mathrm{gX}$ and at least one of $\mathrm{fX}$ or $\mathrm{gX}$ is a complete subset of $\mathrm{X}$. Suppose that there exists an operator $A \in \mathcal{B}(E)$ with $\rho(A)<1$ and $A(C) \subset C$ such that

$$
\mathrm{d}(\mathrm{fu}, \mathrm{fv}) \preceq \mathrm{A}(\mathrm{d}(\mathrm{gu}, \mathrm{g} v)) \quad \text { for all } \mathrm{u}, \boldsymbol{v} \in \mathrm{X} \text { with }(\mathrm{gu}, \mathrm{gv}) \in \mathcal{E} .
$$

If conditions (a)-(d) hold, then $\mathrm{f}$ and $\mathrm{g}$ have a point of coincidence in $\mathrm{X}$. If also $\mathrm{X}$ is $\mathrm{G}$-directed with respect to $\mathrm{f}$ and $\mathrm{g}$, then the point of coincidence is unique. Moreover, if $\mathrm{f}$ and $\mathrm{g}$ are weakly compatible, then they have a unique common fixed point.

\section{Acknowledgment}

The authors extend their appreciation to the International Scientific Partnership Program ISPP at King Saud University for funding this research work through ISPP\#0068.

\section{References}

[1] M. Abbas, V. Rakočević, A. Iqbal, Coincidence and common fixed points of Perov type generalized Ćirić-contraction mappings, Mediterr. J. Math., 13 (2016), 3537-3555. 1

[2] M. Abbas, V. Rakočević, A. Iqbal, Fixed points of Perov type contractive mappings on the set endowed with a graphic structure, Rev. R. Acad. Cienc. Exactas Fís. Nat. Ser. A Mat. RACSAM, (2017), 1-20. 1

[3] J. Ahmad, M. Arshad, C. Vetro, On a theorem of Khan in a generalized metric space, Int. J. Anal., 2013 (2013), 6 pages. 1 
[4] C. D. Aliprantis, R. Tourky, Cones and duality, Graduate Studies in Mathematics, American Mathematical Society, Providence, RI, (2007). 2

[5] A. Azam, M. Arshad, I. Beg, Banach contraction principle on cone rectangular metric spaces, Appl. Anal. Discrete Math., 3 (2009), 236-241. 1

[6] S. Banach, Sur les opérations dans les ensembles abstraits et leur application aux équations intégrales, Fund. Math., 3 (1922), 133-181. 1

[7] A. Branciari, A fixed point theorem of Banach-Caccioppoli type on a class of generalized metric spaces, Publ. Math. Debrecen, 57 (2000), 31-37. 1

[8] M. Cvetković, V. Rakočević, Common fixed point results for mappings of Perov type, Math. Nachr., 288 (2015), 18731890. 3.1

[9] K. Deimling, Nonlinear functional analysis, Springer-Verlag, Berlin, (1985). 2

[10] C. Di Bari, P. Vetro, Common fixed points in generalized metric spaces, Appl. Math. Comput., 218 (2012), $7322-7325.1$

[11] L.-G. Huang, X. Zhang, Cone metric spaces and fixed point theorems of contractive mappings, J. Math. Anal. Appl., 332 (2007), 1468-1476. 1, 2

[12] Z. Kadelburg, S. Radenović, Fixed point results in generalized metric spaces without Hausdorff property, Math. Sci. (Springer), 8 (2014), 8 pages. 1

[13] Z. Kadelburg, S. Radenović, On generalized metric spaces: a survey, TWMS J. Pure Appl. Math., 5 (2014), 3-13. 1

[14] P. Kumam, C. Vetro, F. Vetro, Fixed points for weak $\alpha-\psi$-contractions in partial metric spaces, Abstr. Appl. Anal., 2013 (2013), 9 pages. 4

[15] V. La Rosa, P. Vetro, Common fixed points for $\alpha-\psi-\phi$-contractions in generalized metric spaces, Nonlinear Anal. Model. Control, 19 (2014), 43-54. 1

[16] S. K. Malhotra, S. Shukla, R. Sen, Some fixed point theorems for ordered Reich type contractions in cone rectangular metric spaces, Acta Math. Univ. Comenian. (N.S.), 82 (2013), 165-175. 1

[17] A. I. Perov, On the Cauchy problem for a system of ordinary differential equations, (Russian) Približ. Metod. Rešen. Differencial'. Uravnen. Vyp., 2 (1964), 115-134. 1

[18] A. I. Perov, A. V. Kibenko, On a certain general method for investigation of boundary value problems, (Russian) Izv. Akad. Nauk SSSR Ser. Mat., 30 (1966), 249-264. 1

[19] B. Samet, Discussion on "A fixed point theorem of Banach-Caccioppoli type on a class of generalized metric spaces" by A. Branciari [ MR1771669], Publ. Math. Debrecen, 76 (2010), 493-494. 1

[20] B. Samet, C. Vetro, P. Vetro, Fixed point theorems for $\alpha-\psi$-contractive type mappings, Nonlinear Anal., 75 (2012), 2154-2165. 4

[21] S. Shukla, Reich type contractions on cone rectangular metric spaces endowed with a graph, Theory Appl. Math. Comput. Sci., 4 (2014), 14-25. 1

[22] S. Shukla, S. Balasubramanian, M. Pavlović, A generalized Banach fixed point theorem, Bull. Malays. Math. Sci. Soc., 39 (2016), 1529-1539. 3

[23] P. Vetro, Common fixed points in cone metric spaces, Rend. Circ. Mat. Palermo, 56 (2007), 464-468. 3

[24] C. Vetro, On Branciari's theorem for weakly compatible mappings, Appl. Math. Lett., 23 (2010), 700-705. 1

[25] Y. C. Wong, K. F. Ng, Partially ordered topological vector spaces, Oxford Mathematical Monographs, Clarendon Press, Oxford, (1973). 2

[26] S.-Y. Xu, Ć. Dolićanin, S. Radenović, Some remarks on results of Perov type, J. Adv. Math. Stud., 9 (2016), 361-369. 2.5

[27] P. P. Zabrejko, K-metric and K-normed linear spaces: survey, Fourth International Conference on Function Spaces, Zielona Góra, (1995), Collect. Math., 48 (1997), 852-859. 1 